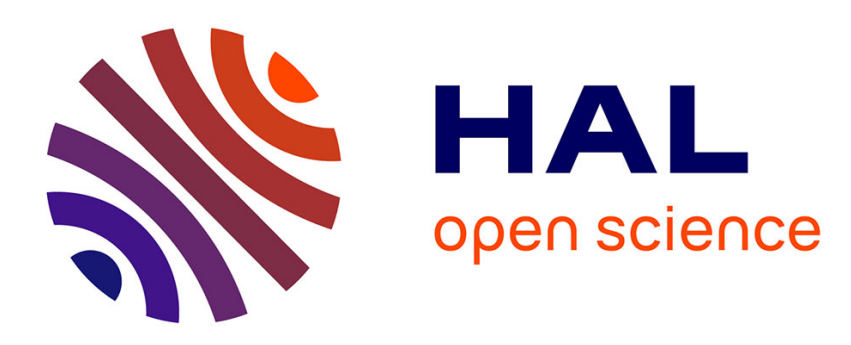

\title{
Solis: a modular, portable, and high-performance 1D semi-conductor device simulator
}

Sidi Ould Saad Hamady

\section{To cite this version:}

Sidi Ould Saad Hamady. Solis: a modular, portable, and high-performance 1D semi-conductor device simulator. Journal of Computational Electronics, 2020, 19 (2), pp.640-647. 10.1007/s10825-02001477-7 . hal-03247058

\section{HAL Id: hal-03247058 \\ https://hal.univ-lorraine.fr/hal-03247058}

Submitted on 2 Jun 2021

HAL is a multi-disciplinary open access archive for the deposit and dissemination of scientific research documents, whether they are published or not. The documents may come from teaching and research institutions in France or abroad, or from public or private research centers.
L'archive ouverte pluridisciplinaire HAL, est destinée au dépôt et à la diffusion de documents scientifiques de niveau recherche, publiés ou non, émanant des établissements d'enseignement et de recherche français ou étrangers, des laboratoires publics ou privés. 


\title{
Solis: a modular, portable and high performance 1D semiconductor device simulator
}

\author{
Sidi Ould Saad Hamady \\ Université de Lorraine, CentraleSupélec, LMOPS, F-57000 Metz, France. \\ Published in Journal of Computational Electronics \\ https://doi.org/10.1007/s10825-020-01477-7
}

\begin{abstract}
This article presents Solis, a new modular, fast and portable one-dimensional (1D) semiconductor device simulator designed and developed particularly for photovoltaic solar cells. Solis is coded in standard C++, runs natively on Windows and Linux and is freely available to download. All the physical models and excitation parameters can be fully controlled using the fast embedded Lua scripting engine. Solis includes a useful set of tools coded in $\mathrm{C}$, such as a code editor, graphical device editor and data plotter. A material parameters database including well-known semiconductors and their alloys is also included. The Solis calculation engine implements the drift-diffusion transport model and takes into account indirect recombination processes (with user defined deep or shallow levels) as well as radiative and Auger recombination. The anode and cathode parameters, including the refractive index, extinction coefficient, recombination speed and barrier height for Schottky contact are fully included. It natively handles spontaneous and piezoelectric polarization, which is key for the development of devices based on wurtzite materials. The values of all the material parameters can be graded arbitrarily in position, offering flexibility for the simulation of heterostructure solar cells. In addition, Solis offers functionality needed by researchers to analyze the simulation results, including graphing (of the band diagram, carrier concentration, ionized dopant and trap concentrations, current voltage, capacitance voltage, quantum efficiency etc.) with complete control of the graph and a useful mathematical console. Solis simulation results for a solar cell using Earth-abundant elements are presented herein. One of the main issues in the development of such solar cells, viz. the inhomogeneity due to phase separation in the absorber, is highlighted.
\end{abstract}

Keywords: Solis, Simulation, Semiconductor devices, Scripting, Design, Portable, Solar cell, Heterostructure

\section{INTRODUCTION}

Numerical simulation is an important step in the design and elaboration of novel photovoltaic solar cells. Over the years, several noncommercial one-dimensional device simulators have been developed [1-3], contributing to the development of the photovoltaic industry. These tools are not portable and can hardly be upgraded or extended; For example, PC1D [1], the main one-dimensional simulator, was developed in the 1990s for the Windows operating system and uses the nonportable Microsoft Foundation Class (MFC) library with a code basis mixing calculation algorithms and user interface routines. It has been widely used in the photovoltaic community, with the last official version released in June 2003 and updated in 2008 with the release of its source code. ${ }^{1}$ SCAPS [3], another popular one-dimensional simulator with a large number of features and regular updates, uses the proprietary National Instruments LabWindows CVI development framework used in virtual instrumentation and running only on Windows. The last official version of AMPS-1D [2], one of the three main noncommercial device simulators, dates from 1997, and runs natively only on Windows 95 up to XP (requiring a Windows 95 up to XP virtual machine to run on Windows $7 / 8 / 10)^{2}$

\footnotetext{
${ }^{1}$ An unofficial release of PC1D named PC1D-mod was developed with minor changes but unfortunately removing the ability to handle heterostructures.

${ }^{2}$ As for PC1D, there is an unofficial release of AMPS-1D named wxAMPS with changes mainly related to the user interface.
}

Secondly, the existing tools, mainly the three most widely used ones presented above, do not use some paradigms in computer science related, for instance, to modularity, portability, interoperability, and versatility. None of these free simulators includes a scripting language like in commercial simulators. ${ }^{3}$ SCAPS uses a mechanism tightly linked to the user interface to automate user actions, without being a scripting language. Including a scripting language is nowadays necessary to extend possibilities of the simulator for the development of, for example, specific physical models, graded devices, complex nonhomogeneous structures, or optimization of material parameters. On the other hand, these tools do not separate the user interface from the simulation engine; they are mixed by design. The nonmodularity, lack of separation between the user interface and backend simulation engine, and the wide use of nonportable proprietary formats and frameworks automatically imply the features underlined here and highlight the limitations now faced by these existing tools.

Thirdly, Solis includes features that are not included in the mentioned 1D simulators. It can handle the spontaneous and piezoelectric polarizations, an important feature for wurtzite semiconductor materials such as gallium nitride and related alloys. In addition, it handles Schottky contacts and heterostructures in a more comprehensive way and has the capability to simulate graded semiconductor devices easily.

These facts justify the need for a new simulator that takes

\footnotetext{
${ }^{3}$ For example, the commercial reference simulator Atlas ${ }^{\circledR}$ from Silvaco ${ }^{\circledR}$ includes a $\mathrm{C}$ interpreter that can be used to extend it and develop custom physical models.
} 
into account, at each step, the criteria of portability, modularity, versatility, and performance which are now becoming crucial considering the development of heterogeneous computing platforms and the central role of simulation in the development of novel devices. I therefore developed Solis, a new 1D device simulator particularly adapted for solar cell simulation. Solis runs natively on Windows (from XP to 10) or Linux (Debian, CentOS, Ubuntu, etc.) and is easily portable to other platforms including, but not limited to, Android and macOS. It is freely available to download from the author's website $^{4}$ [4]. Its standard C++ coded simulation engine, optimized at each step of its development process, ensures high performance while retaining complete portability. The embedded fast just in time Lua scripting engine gives the user control over every aspect of the simulation. Solis implements the drift-diffusion model by solving the Poisson-continuity equations, using both the coupled Newton method and the iterative Gummel decoupled method. It can also calculate the band diagram of any heterostructure (using the given database parameters or adding new material parameters), the potential, field and carrier concentration distributions, the currentvoltage characteristic in the dark and under illumination (with a monochromatic, AM1.5 or any user defined spectrum), the capacitance-voltage characteristic, the external quantum efficiency, etc. It takes into account various recombination processes and can handle both nonideal ohmic and Schottky contacts, and can also include spontaneous and piezoelectric polarizations in wurtzite III-N materials. Solis is complementary to some very new tools such as Simudo [5] and Solcore [6]. Since Solis is fully implemented in C++ and offers high performance while being fully extensible through the integrated scripting language, it is more suitable for 1D simulation and numerical optimization while these Python tools are suitable for specific simulations including, e.g., quantum effects or intermediate-band materials.

\section{MODELING, NUMERICAL METHODS AND IMPLEMENTATION}

The Solis simulation engine uses the drift-diffusion model, which is the de facto standard physical model used to describe and calculate the electrical properties of semiconductor devices [7]. Solis implements both the coupled Newton method and the decoupled Gummel method to solve the model equations.

\footnotetext{
${ }^{4}$ The latest Solis release (extracted total size less than $10 \mathrm{MB}$ ) is available to download:

http://www.hamady.org/download/solis_windows_64bit.zip (for Windows XP/Vista/7/8/10 64 bit)

http://www.hamady.org/download/solis_linux_64bit.tgz (for Linux (Debian, CentOS, Ubuntu, etc.) 64 bit).

The Solis user manual is also available to download here: http://www.hamady.org/download/solis_simulator.pdf
}

\section{A. Drift-diffusion model}

The drift-diffusion model is composed of the Poisson equation and the two current continuity equations, as shown in onedimensional $\mathrm{z}$ and normalized form in Eq. 1. The normalization factor in the Poisson equation is taken to be equal to unity, using the Debye length as the position normalization parameter.

$$
\begin{aligned}
\frac{\mathrm{d}}{\mathrm{d} z}\left(\varepsilon(z) \frac{\mathrm{d} V(z)}{\mathrm{d} z}+P(z)\right) & =n(z)-p(z) \\
& +N_{A}^{-}(z)-N_{D}^{+}(z) \\
& +\sum_{t} N_{t A}^{-}(z)-\sum_{t} N_{t D}^{+}(z) \\
\frac{\mathrm{d} J_{n}}{\mathrm{~d} z} & =-\mathrm{GR}(z) / \gamma^{2} \\
\frac{\mathrm{d} J_{p}}{\mathrm{~d} z} & =+\mathrm{GR}(z) / \gamma^{2} \\
J_{n}(z) & =-n(z) \mu_{n} \frac{d \phi_{n}(z)}{\mathrm{d} z} \\
J_{p}(z) & =+p(z) \mu_{p} \frac{d \phi_{p}(z)}{\mathrm{d} z}
\end{aligned}
$$

with the electrostatic potential $\mathrm{V}$, the spontaneous and piezoelectric polarization $\mathrm{P}$ (if any), the permittivity $\varepsilon$, the free electron concentration $\mathrm{n}$, the free hole concentration $\mathrm{p}$, the ionized donor $\mathrm{N}_{\mathrm{D}}^{+}$and ionized acceptor $\mathrm{N}_{\mathrm{A}}^{-}$concentration, and the ionized donor- $\mathrm{N}_{\mathrm{tD}}^{+}$and acceptor-like trap $\mathrm{N}_{\mathrm{tA}}^{-}$concentration. The concentrations can vary with the position coordinate $\mathrm{z}$. $\mathrm{J}_{\mathrm{n}}$ and $\mathrm{J}_{\mathrm{p}}$ are the electron and hole currents, and GR the net generation term. $\gamma^{2}$ is a normalization parameter (the continuity factor shown in Table I). $\mu_{\mathrm{n}}$ and $\mu_{\mathrm{p}}$ are the electron and hole mobilities, $\phi_{\mathrm{n}}$ and $\phi_{\mathrm{p}}$ are the normalized quasi-Fermi levels.

All the parameters in these equations are dimensionless, i.e. normalized, using the normalization parameters defined in Table I. The currents are expressed in terms of quasi-Fermi levels for simplicity, and include both drift and diffusion terms. The net generation term GR(z) includes light-induced carrier generation (photogeneration) and the various recombination phenomena, including nonradiative Shockley-Read-Hall (SRH), radiative and Auger mechanisms.

The electron and hole concentrations are given by

$$
\begin{aligned}
& n(z)=n_{i} e^{\left(+V(z)+V_{n}(z)-\phi_{n}(z)\right)} \\
& p(z)=n_{i} e^{\left(-V(z)+V_{p}(z)+\phi_{p}(z)\right)}
\end{aligned}
$$

where $n_{i}$ is the intrinsic carrier concentration. $V_{n}$ and $V_{p}$ are the band parameters for heterostructures and nonuniform graded materials (i.e. for homostructures $V_{n}=V_{p}=0$ ). 


\begin{tabular}{ll}
\hline Parameter & Normalization factor \\
\hline \hline Permittivity & $\varepsilon_{0}$ \\
\hline Potential & Thermal voltage, $\mathrm{kT} / \mathrm{q}$ \\
\hline Energy & Thermal energy, $\mathrm{kT}$ \\
\hline Carrier concentration & $\mathrm{N}=10^{22} \mathrm{~m}^{-3}$ \\
\hline Space & Debye length, $\mathrm{L}=\sqrt{\varepsilon_{0} \mathrm{kT} /\left(\mathrm{q}^{2} \mathrm{~N}\right)}$ \\
\hline Mobility & $\mu_{0}=0.1 \mathrm{~m}^{2} / \mathrm{V}-\mathrm{s}$ \\
\hline Recombination & $\beta_{0}=10^{-20} \mathrm{~m}^{3} / \mathrm{s}$ \\
\hline Continuity factor & $\gamma^{2}=\mu_{0} \mathrm{kT} /\left(\beta_{0} \mathrm{qNL}{ }^{2}\right)$ \\
\hline
\end{tabular}

TABLE I: Normalization parameters used for the drift-diffusion model.

The net generation term $\mathrm{GR}(\mathrm{z})$ is expressed as:

$$
\begin{aligned}
G R(z) & =G(z)-R_{D}(z)-R_{I}(z)-R_{A}(z) \\
G(z) & =\int_{\lambda} \alpha(\lambda, z) \phi(\lambda) \exp (-\alpha(\lambda, z) z) d \lambda \\
R_{D} & =\beta\left(n p-n_{i}^{2}\right) \\
R_{I} & =\sum_{t} \frac{\left(n p-n_{i}^{2}\right)}{\tau_{p}\left(n+n_{i} \exp \left(E_{t}-E_{i}\right)\right)+\tau_{n}\left(p+n_{i} \exp \left(-E_{t}+E_{i}\right)\right)} \\
R_{A} & =\left(n p-n_{i}^{2}\right)\left(C_{n} n+C_{p} p\right)
\end{aligned}
$$

where $G(z)$ is the photogeneration rate, $R_{D}(z)$ is the direct (radiative) recombination rate, $\mathrm{R}_{\mathrm{I}}(\mathrm{z})$ is the $\mathrm{SRH}$ recombination rate and $R_{A}(z)$ is the Auger recombination rate. $R_{I}(z)$ account for dopants and traps (where $\mathbf{t}$ index stands for trap), each described by an activation energy $E_{t}$ and electron and hole lifetimes $\tau_{\mathrm{n}}$ and $\tau_{\mathrm{p}}$. $\mathrm{E}_{\mathrm{i}}$ is the intrinsic Fermi level. $\alpha(\lambda)$ is the absorption coefficient, varying with the light wavelength $\lambda$, and $\phi$ the incident light flux. $\beta$ is the direct recombination coefficient. $C_{n}$ and $C_{p}$ are the Auger coefficients for electrons and holes, respectively.

\section{B. Solving methods and Solis implementation}

The drift-diffusion model as defined in Eq. 1 is solved to determine the potential $V(z)$ and the quasi-Fermi levels $\phi_{\mathrm{n}}(\mathrm{z})$ and $\phi_{\mathrm{p}}(\mathrm{z})$. The other quantities are then calculated using these fundamental parameters. The Scharfetter-Gummel discretization scheme [8] is used to ensure the numerical stability needed in this partial differential equation (PDE) system. The discretized PDE system is then solved using two methods implemented in Solis, giving complete flexibility:

- The Gummel iterative decoupled method. In this method, the three equations are solved iteratively until convergence: Starting with initial guess vectors $\mathrm{V}(\mathrm{z})$, $\phi_{\mathrm{n}}(\mathrm{z})$, and $\phi_{\mathrm{p}}(\mathrm{z})$, Solis solves the discretized Poisson system, using the Newton-Raphson method and determines the new value of the potential vector $\mathrm{V}(\mathrm{z})$, which is then used to solve the electron continuity equation and determine the $\phi_{\mathrm{n}}(\mathrm{z})$ vector. $\mathrm{V}(\mathrm{z})$ and $\phi_{\mathrm{n}}(\mathrm{z})$ are then used to solve the hole continuity equation to get the $\phi_{p}(z)$ vector and the process iterates until convergence. A damping strategy is implemented is Solis to ensure convergence in most cases since this method can converge slowly for problems where the three driftdiffusion equations are highly coupled.

- The Newton method solves the full discretized nonlinear drift-diffusion PDE system simultaneously using the Newton-Raphson method. This method is suitable for highly coupled cases and can converge fast providing that a good starting solution is given. Solis provides a starting point for the solver to speed up the convergence, and can also use the Gummel method to refine the initial guess.

Solis can handle both Dirichlet and Neumann boundary conditions. The device contacts can be nonideal ohmic or rectifying (Schottky). The numerical parameters, such as the starting initial guess, the damping parameter and tolerance, and the boundary conditions, can be set by the user to handle specific structures, even if Solis automatically adapts these parameters for convergence.

As underlined above, Solis has been developed with modularity, versatility, and performance as the main criteria and guidelines. Its general architecture is shown in Fig. 1 and its main components are:

- The simulator driver, soliscomp.exe (or soliscomp under Linux), controlling the simulator engine. The driver and the simulation engine are developed in standard $\mathrm{C}++$ implementing the numerical methods described above and handling the whole simulation process: input file format and device definition, memory management, data input and output and solving procedures. The simulator driver also controls the embedded Lua scripting engine that can be used to implement user-defined physical models and parameters, permitting advanced control of the simulation process. The simulator driver is a command line executable with a device input in a simple and clean text format. It can be easily controlled and automated by any language (and thus the embedded Lua interpreter), giving the user unprecedented flexibility and control over the simulation.

- A graphical device editor, solisdevice.exe (or solisdevice under Linux), implemented in C. This tool provides an easy-to-use graphical frontend to Solis.

- An advanced code editor, solisedit.exe (or solisedit under Linux), implemented in $\mathrm{C}$, to edit the device structure using the straightforward Solis syntax. This editor offers all functionality found in modern editors and can be used as a general code editor. 


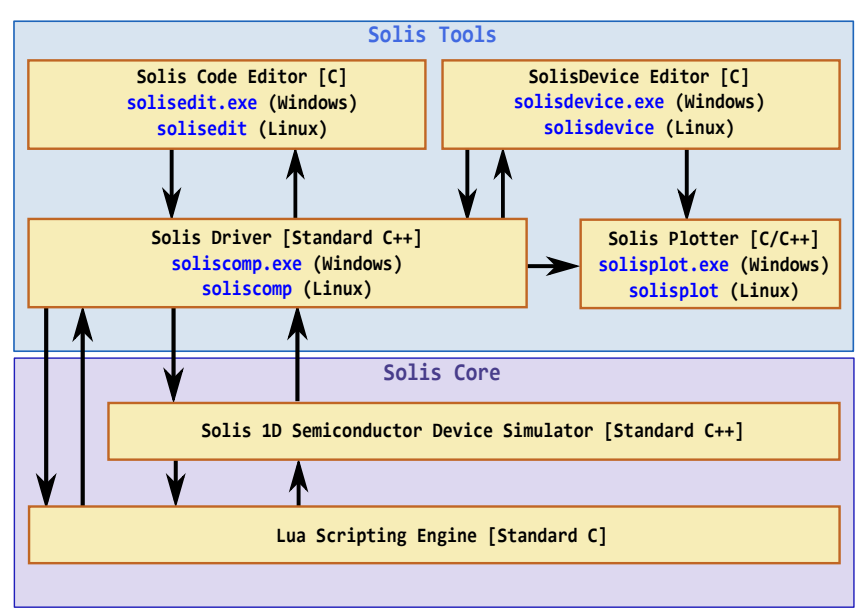

FIG. 1: The Schematic Solis architecture. The core part of

Solis is entirely coded in standard C++ (the Lua engine being coded in standard C) and is easily portable to almost all operating systems, in addition to the natively supported Windows and Linux systems. The Solis code editor, device editor, and data plotter are developed in C and use the IUP GUI toolkit coded in C [9] and the Scintilla component coded in $\mathrm{C}++[10]$.

- A data plotter, solisplot.exe (under Windows) or solisplot (under Linux), implemented in C and C++. This tool is used by Solis to plot the simulation results but could also be used as a standalone data plotter.

- An advanced scientific calculator, soliscalc, implemented in $\mathrm{C}$.

The size of the whole Solis distribution, including documentation and examples, is less than $10 \mathrm{MB}$.

These tools are independent and can be used in a complementary way; For example, solisdevice can be used to perform simulations in a complete graphical environment. To develop a complete simulation framework, with custom physical models and optimization scripts, one can use solisedit to achieve full control over the code. These tools are, by design, compatible; e.g., an input file created with the code editor can be open/modified by the graphical device editor, and vice-versa.

The Solis input format is an easy-to-use plain text format with simple syntax and some useful features such as variable definition and a mathematical parser.

A user manual, describing the underlying physics, the input syntax, the models used, the implementation, and the software tools, is provided with the Solis distribution.

\section{AN APPLICATION TO THE STUDY OF CZTS SOLAR CELLS}

Next-generation thin-film solar cells face, among other challenges, two critical issues: their overall cost and environmental impact. The overall cost is affected by the lack
AM1.5 Light

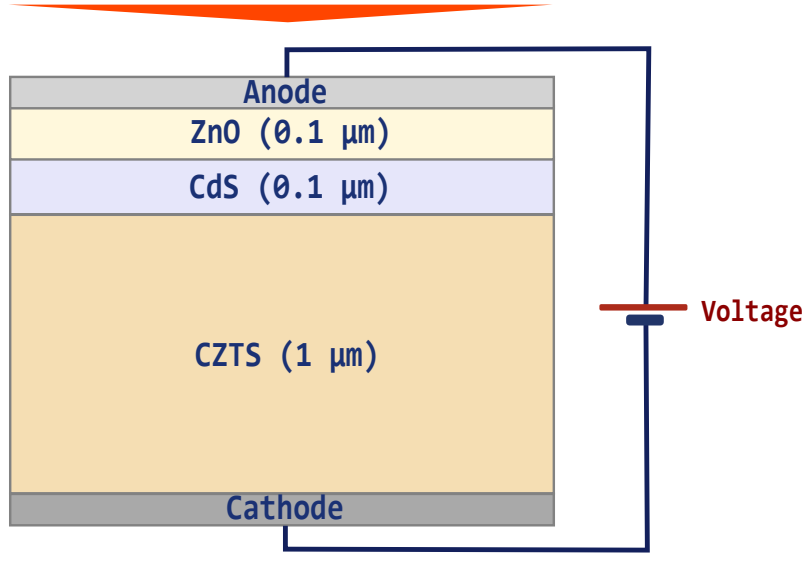

FIG. 2: The $\mathrm{ZnO} / \mathrm{CdS} / \mathrm{CZTS}$ solar cell structure. The $\mathrm{ZnO}$ and $\mathrm{CdS}$ layers are n-doped with a donor concentration of $10^{18} \mathrm{~cm}^{-3}$. The CZTS p-doping concentration is equal to $10^{16} \mathrm{~cm}^{-3}$.

of abundance of the chemical elements contained in the active layers and the elaboration techniques used to fabricate such solar cells. On the other hand, environmental impact must take into account, for example, the toxicity of the chemical elements used in the elaboration process and the recycling procedure. One of the main compound semiconductors used in the thin-film solar cell technology is copper indium gallium selenide $\mathrm{CuIn}_{1-\mathrm{x}} \mathrm{Ga}_{\mathrm{x}} \mathrm{Se}_{2}$ (CIGS) with the record efficiency recently reaching $22 \%$ [11]. While mature, CIGS technology is affected by the use of indium, costly and rare element, that is already widely used in screen manufacturing [12]. Other technologies that are far less mature than CIGS are also affected by their use of indium, e.g., those based on the potentially promising indium gallium nitride ( $\mathrm{InGaN})$ alloy $[13,14]$. To address this issue, several materials using Earth-abundant and nontoxic elements have been developed. Among these materials, copper zinc tin sulfide $\mathrm{Cu}_{2} \mathrm{ZnSnS}_{4}$ (CZTS) alloys (and, to a lesser extent, copper zinc tin selenide $\mathrm{Cu}_{2} \mathrm{ZnSnSe}_{4}$ (CZTSe)), are become promising materials for use in thin-film solar cells $[15,16]$. The record photovoltaic efficiency obtained using these new materials, $12.6 \%$ [16], while below the CIGS efficiency, is higher than that of the well-established amorphous silicon solar cells. CZTS has the potential to reach the CIGS level if some issues can be addressed. Some of these issues are related to phase separation in these complex alloys.

The next sections present results for a $\mathrm{ZnO} / \mathrm{CdS} / \mathrm{CZTS}$ solar cell, firstly without phase separation and, secondly, with a phase separation simulated using the capability of Solis to handle arbitrary position-varying physical properties.

\section{A. The single-phase CZTS solar cell}

Figure 2 shows the simulated structure with the $\mathrm{ZnO}$ window, the CdS buffer layer, and the stoichiometric single-phase 


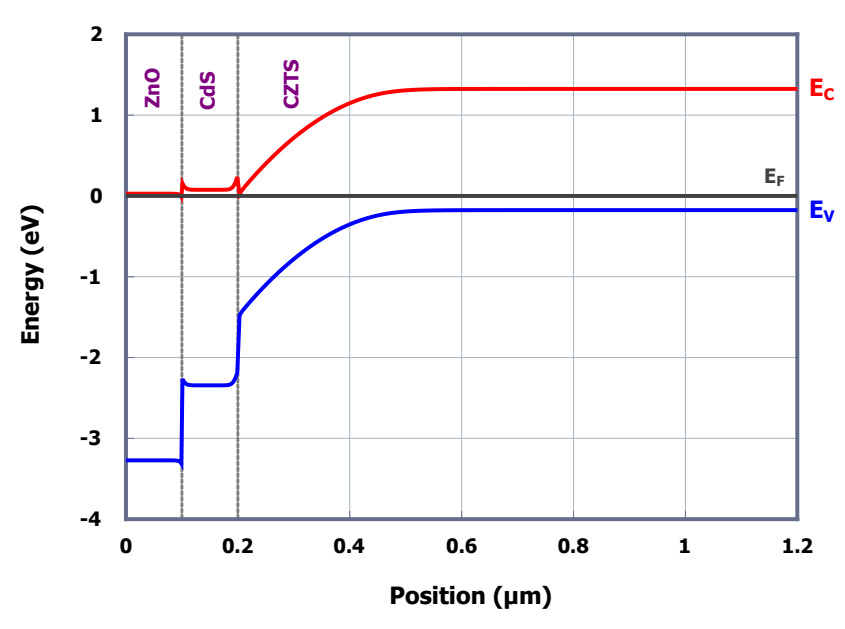

FIG. 3: The ZnO/CdS/CZTS band diagram at thermodynamic equilibrium.

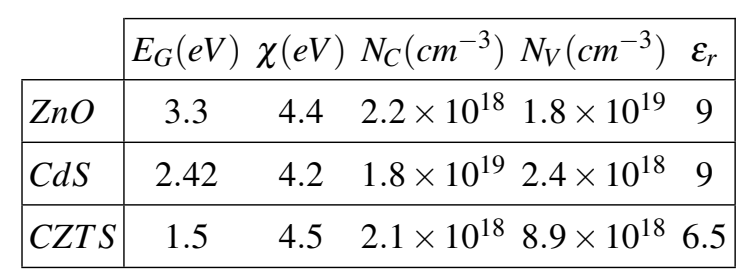

TABLE II: The basic parameters of CZTS, CdS, and ZnO used in the simulation. $\mathrm{E}_{\mathrm{G}}$ is the bandgap, $\chi$ is the electron affinity, $\mathrm{N}_{\mathrm{C}}$ and $\mathrm{N}_{\mathrm{V}}$ are the density of states in the conduction and valence band, respectively, and $\varepsilon_{\mathrm{r}}$ is the relative permittivity. The direct and Auger recombination parameters are set to $\beta=10^{-12} \mathrm{~cm}^{3} / \mathrm{s}$ and $\mathrm{C}_{\mathrm{n}}=\mathrm{C}_{\mathrm{p}}=10^{-30} \mathrm{~cm}^{6} / \mathrm{s}$ respectively. The experimental data are taken from $[18,19]$.

CZTS absorber, while Fig. 3 plots the band diagram simulated by Solis at thermodynamic equilibrium. Table II presents the basic CZTS, CdS, and ZnO parameters used in the simulations. The CZTS extinction coefficient varies with the the photon energy according to the standard formula in Eq. 4.

$$
k(E)=k_{o}\left(\frac{2 E_{G}}{E}\right)\left(\frac{E-E_{G}}{E_{G}}\right)^{\frac{1}{2}}
$$

where $\mathrm{E}_{\mathrm{G}}$ is the CZTS bandgap, $\mathrm{E}$ the photon energy and $\mathrm{k}_{\mathrm{o}}$ the extinction coefficient at $\mathrm{E}=2 \times \mathrm{E}_{\mathrm{G}}$, taken to be equal to 0.2 [17].

The used AM1.5 solar spectrum is the ASTM G173-03 reference spectrum taken from NREL [20].

Figure 4 shows the current-voltage characteristic of the homogeneous CZTS solar cell obtained using Solis and that simulated using the Atlas ${ }^{\circledR}$ tool from Silvaco ${ }^{\circledR}$. The obtained photovoltaic efficiency of $\approx 19.2 \%$ is close to the highest reported value for a CIGS solar cell [11] and highlights the potential of this alloy as an alternative to CIGS. Further optimization, taking the doping and thickness values as param-

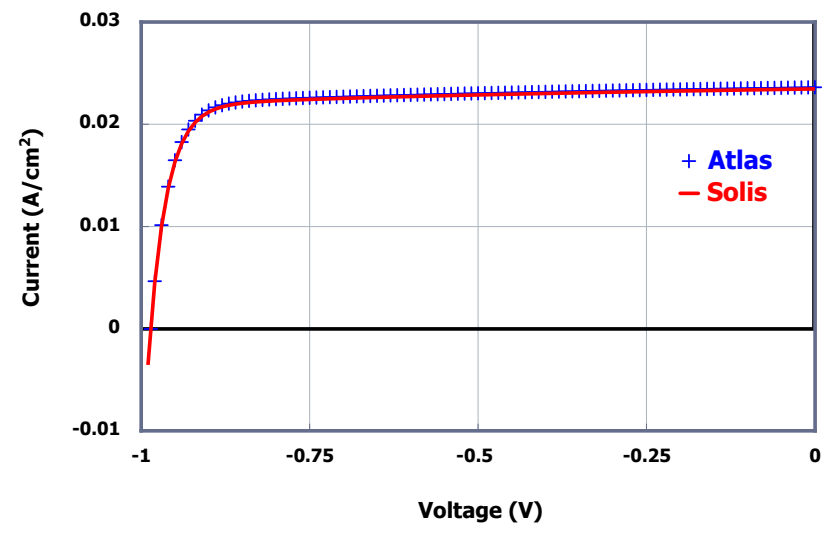

FIG. 4: The current-voltage characteristic of the single phase CZTS solar cell under the illumination by the AM1.5 spectrum. The voltage is applied to the top contact of the n-p structure. The symbols indicates the characteristic simulated using Atlas ${ }^{\circledR}$ from Silvaco ${ }^{\circledR}$, while the straight line shows that simulated by Solis. The photovoltaic performance obtained by Solis and Atlas ${ }^{\circledR}$ is presented in Table III.

\begin{tabular}{|l|cccc|}
\cline { 2 - 5 } \multicolumn{1}{c|}{} & \multicolumn{1}{c}{$\eta(\%)$} & $\mathrm{J}_{\mathrm{SC}}\left(\mathrm{A} / \mathrm{cm}^{2}\right)$ & $\mathrm{V}_{\mathrm{OC}}(\mathrm{V})$ & $\mathrm{FF}(\%)$ \\
\hline Solis & 19.263 & 0.0234 & 0.985 & 82.73 \\
\hline Atlas $^{\circledR}$ & 19.260 & 0.0236 & 0.986 & 82.77 \\
\hline
\end{tabular}

TABLE III: The photovoltaic performance obtained using Solis and Atlas ${ }^{\circledR}$ for the single-phase CZTS solar cell illuminated with an integrated AM1.5 power of $0.099271 \mathrm{~W} / \mathrm{cm}^{2} . \eta$ is the efficiency, $\mathrm{J}_{\mathrm{SC}}$ the short-circuit current, $\mathrm{V}_{\mathrm{OC}}$ the open-circuit voltage and FF the fill factor.

eters, would give the absolute optimum efficiency using, for example, the method presented in Ref. [21]. Another issue with the CZTS solar cell structure is the use of $\mathrm{CdS}$, which contains cadmium. This could be replaced with $\operatorname{In}_{2} \mathrm{~S}_{3}$ [22] or, more consistently, with $\mathrm{ZnS}$ [23], containing Eath-abundant elements.

\section{B. The multiple-phase CZTS solar cell}

In the complex CZTS quaternary alloy, phase separation is usually observed, with evidence of two phases: $\mathrm{ZnS}$ and $\mathrm{Cu}_{2} \mathrm{SnS}_{3}$, in addition to the $\mathrm{Cu}_{2} \mathrm{ZnSnS}_{4}$ phase [24]. The presence of $\mathrm{ZnS}$ is also evidenced between the CZTS absorber and the bottom molybdenum contact [25]. The ability of a simulator to handle any position-varying semiconductor parameters is key to the study and evaluation of the impact of phase separation on the performance of the solar cell. Using Solis, one can set any material graded profile, using the embedded scripting language, and simulate the phase separation in CZTS. To capture the real material properties precisely, the profile of the position-varying parameters (bandgap, affinity, permittiv- 


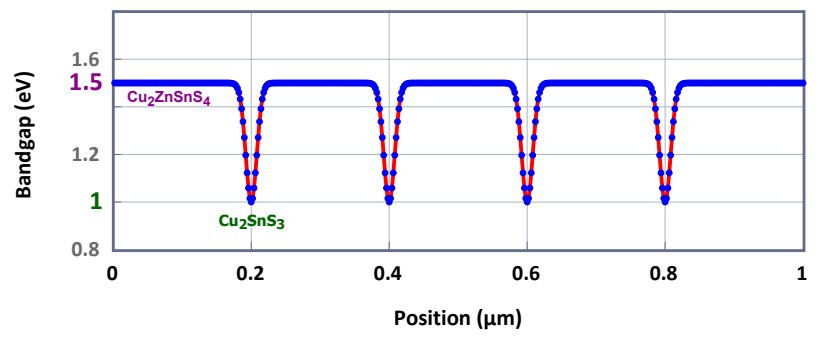

FIG. 5: The periodic inverted Gaussian profile used to model the variation of the bandgap with position in the CZTS alloy based on the $\mathrm{Cu}_{2} \mathrm{SnS}_{3} / \mathrm{Cu}_{2} \mathrm{ZnSnS}_{4}$ phase separation fraction. The bandgap of $\mathrm{Cu}_{2} \mathrm{ZnSnS}_{4}$ is $\approx 1.5 \mathrm{eV}$, while the bandgap of $\mathrm{Cu}_{2} \mathrm{SnS}_{3}$ is $\approx 1 \mathrm{eV}$. The fraction between the secondary $\mathrm{Cu}_{2} \mathrm{SnS}_{3}$ phase and the main $\mathrm{Cu}_{2} \mathrm{ZnSnS}_{4}$ phase is $\zeta=0.16$. The same type of function is used for the other parameters, viz. the affinity, permittivity, and density of states.

\begin{tabular}{|lccccc|}
\hline$\eta(\%)$ & $\mathrm{J}_{\mathrm{SC}}\left(\mathrm{A} / \mathrm{cm}^{2}\right)$ & $\mathrm{V}_{\mathrm{OC}}(\mathrm{V})$ & $\mathrm{FF}(\%)$ & $\mathrm{R}_{\mathrm{S}}\left(\Omega . \mathrm{cm}^{2}\right)$ & $\mathrm{R}_{\mathrm{P}}\left(\Omega . \mathrm{cm}^{2}\right)$ \\
\hline 12.53 & 0.0213 & 0.777 & 75.00 & 2.2 & 139 \\
\hline
\end{tabular}

TABLE IV: The photovoltaic performance obtained by Solis for the multiple phase CZTS solar cell with $\zeta=0.16$ when illuminated with an integrated AM1.5 power of

$0.099271 \mathrm{~W} / \mathrm{cm}^{2} . \eta$ is the efficiency, $\mathrm{J}_{\mathrm{SC}}$ is the short-circuit current, $\mathrm{V}_{\mathrm{OC}}$ is the open-circuit voltage, FF is the fill factor,

$\mathrm{R}_{\mathrm{S}}$ the series resistance, and $\mathrm{R}_{\mathrm{P}}$ is the shunt resistance.

ity, density of states, etc.) can be continuous and inspired by experience; For instance, an abrupt square profile can be used, and is in fact equivalent to defining a multilayer structure (or superlattice), but this is not well adapted to a material showing phase separation. A continuous profile with no abrupt changes in the physical parameters is more well adapted to the realistic simulation of multiphase alloys. Such a smoothed profile is modeled here using a periodic inverted Gaussian function, as plotted in Fig. 5 .

The analysis presented here is limited to the two phases usually observed in this material: $\mathrm{Cu}_{2} \mathrm{ZnSnS}_{4}$ with a bandgap of $\approx 1.5 \mathrm{eV}$, and $\mathrm{Cu}_{2} \mathrm{SnS}_{3}$ with a bandgap of $\approx 1 \mathrm{eV}$. In the chosen profile, the ratio between the Gaussian full width at half maximum (FWHM) and the total period represents the fraction between the secondary $\mathrm{Cu}_{2} \mathrm{SnS}_{3}$ phase and the main $\mathrm{Cu}_{2} \mathrm{ZnSnS}_{4}$ phase. This ratio $\zeta$ is equal to zero for a homogeneous CZTS layer, as presented in Sect. III A, and is now increased up to 0.4 .

Figure 6 shows the band diagram simulated by Solis at thermodynamic equilibrium, for the multiple phase CZTS absorber with a phase ratio $\zeta=0.16$.

The photovoltaic performance obtained by Solis for the multiple phase CZTS solar cell with $\zeta=0.16$ is presented in Table IV. For comparison, the performance for the homogeneous CZTS absorber is shown in Fig. 4.

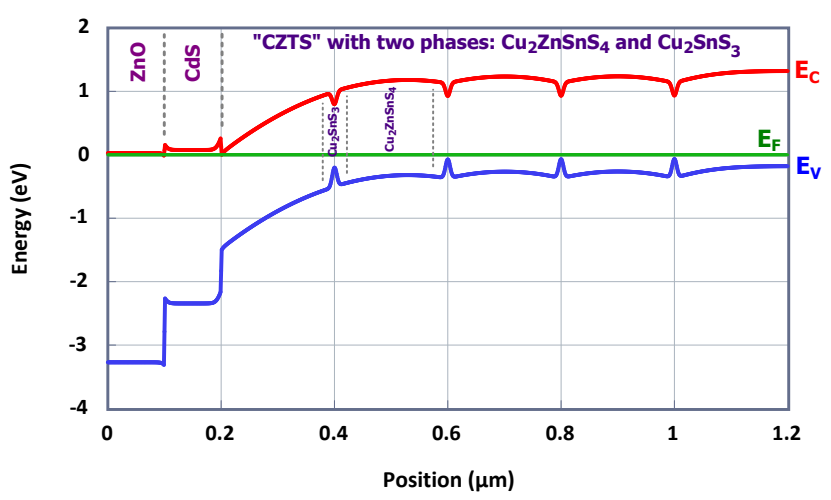

FIG. 6: The ZnO/CdS/CZTS band diagram at thermodynamic equilibrium for the multiple-phase CZTS absorber.

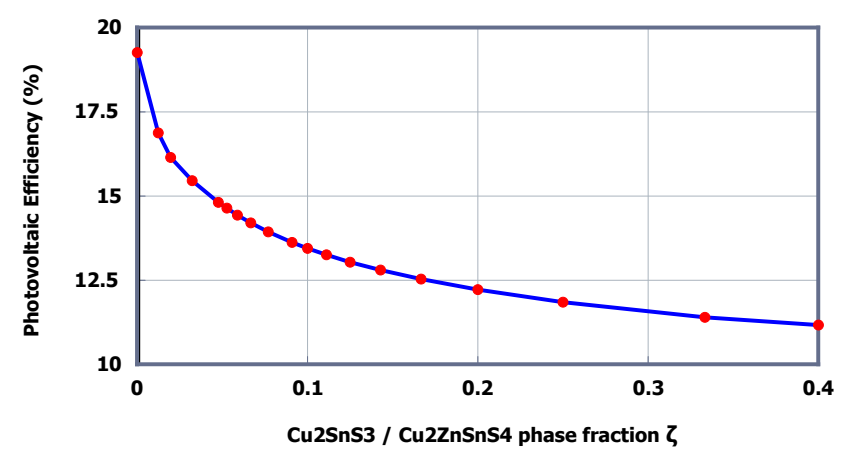

FIG. 7: The variation of efficiency of the multiple-phase CZTS solar cell with $\zeta$, the fraction between the secondary

$\mathrm{Cu}_{2} \mathrm{SnS}_{3}$ phase and the main $\mathrm{Cu}_{2} \mathrm{ZnSnS}_{4}$ phase in the absorber.

The phase separation dramatically impacts the efficiency of the solar cell, even for a relatively low $\zeta$ ratio, and the efficiency $\approx 12 \%$ found here approaches the experimental record [16]. To illustrate this behavior, Fig. 7 shows the variation of the photovoltaic efficiency with respect to $\zeta$, the fraction between the secondary $\mathrm{Cu}_{2} \mathrm{SnS}_{3}$ phase and the main $\mathrm{Cu}_{2} \mathrm{ZnSnS}_{4}$ phase. The efficiency decreases rapidly to reach $11 \%$ for a fraction of 0.4. In this complex heterostructure, with continuously varying CZTS absorber parameters, the change in the electrical field profile causes a decrease in the open-circuit voltage and the accompanying impact on the photogenerated carrier collection decreases the short-circuit current. Other models, including e.g. a random distribution and interface related inhomogeneities, could be used to simulate the effect of phase separation. For more complex structures, a more specific and comprehensive approach, in two dimensions (2D) or even three dimensions (3D), should be used to capture the effect of phase separation in the solar cell absorber more precisely. 


\section{CONCLUSIONS}

A new modular, portable and high-performance 1D semiconductor device simulator is presented. This free simulator, Solis, is developed in standard $\mathrm{C}++$, runs natively on Windows and Linux and is portable to other platforms. It includes a fast scripting engine permitting the development of custom physical models and excitation parameters with a clean and simple language and simulation input. An application to the study of a CZTS heterostructure solar cell is presented, taking into account phase separation in the absorber, modeled using the capability of Solis to handle arbitrary position-varying pa- rameters. The Solis simulator is continuously updated and will include in the coming release a coherent optical propagation model and the possibility to simulate the admittance spectroscopy characteristic, while the transient simulation functionality will be included if it turns out to be important for emerging photovoltaic technologies. Tunneling through the anode Schottky contact, for Schottky solar cells or detectors, is already implemented and will be included in the next release.

\section{CONFLICT OF INTEREST}

The author declares no conflicts of interest.
[1] Basore P and Clugston D 1996 PC1D version 4 for Windows: from analysis to design Photovoltaic Specialists Conference, 1996., Conference Record of the Twenty Fifth IEEE (IEEE) pp 377-381

[2] Zhu H, Kalkan A, Hou J and Fonash S 1999 Applications of AMPS-1D for solar cell simulation AIP Conference Proceedings vol 462 (AIP) pp 309-314

[3] Burgelman M, Nollet P and Degrave S 2000 Thin Solid Films $361527-532$

[4] Ould Saad Hamady S 2010- Software for Photovoltaics http://www.hamady.org/photovoltaics.html

[5] Dumitrescu E, Wilkins M and Krich J 2019 Journal of Computational Electronics 19

[6] Alonso-Álvarez D, Wilson T, Pearce P, Führer M, Farrell D and Ekins-Daukes N 2018 Journal of Computational Electronics 17 1099-1123

[7] Selberherr S 2012 Analysis and simulation of semiconductor devices (Springer Science \& Business Media)

[8] Scharfetter D and Gummel H 1969 IEEE Transactions on electron devices 16 64-77

[9] 2017 The IUP GUI toolkit, (C) 1994-2017 Tecgraf/PUC-Rio

[10] 2016 The Scintilla Component, (C) 1998-2016 Neil Hodgson

[11] Kamada R, Yagioka T, Adachi S, Handa A, Tai K, Kato T and Sugimoto H 2017 New world record $\mathrm{Cu}(\mathrm{In}, \mathrm{Ga})(\mathrm{Se}, \mathrm{S})_{2}$ thin film solar cell efficiency beyond 22\% Photovoltaic Specialist Conference (PVSC), 2017 IEEE 44th (IEEE) pp 1-5

[12] Woodhouse M, Goodrich A, Margolis R, James T, Lokanc M and Eggert R 2012 Supply-chain dynamics of tellurium, indium and gallium within the context of pv module manufacturing costs Photovoltaic Specialists Conference (PVSC), Volume
2, 2012 IEEE 38th (IEEE) pp 1-6

[13] Jani O, Ferguson I, Honsberg C and Kurtz S 2007 Applied Physics Letters 91132117

[14] Ould Saad Hamady S, Adaine A and Fressengeas N 2016 Materials Science in Semiconductor Processing 41 219-225

[15] Jiang M and Yan X $2013 \mathrm{Cu}_{2} \mathrm{ZnSnS}_{4}$ thin film solar cells: present status and future prospects Solar Cells-Research and Application Perspectives (InTech)

[16] Wang W, Winkler M, Gunawan O, Gokmen T, Todorov T, Zhu Y and Mitzi D 2014 Advanced Energy Materials 4

[17] Jiang M, Li Y, Dhakal R, Thapaliya P, Mastro M, Caldwell J, Kub F and Yan X 2011 Journal of Photonics for Energy 1 019501

[18] Madelung O 2012 Semiconductors: data handbook (Springer Science \& Business Media)

[19] Simya O, Mahaboobbatcha A and Balachander K 2015 Superlattices and Microstructures 82 248-261

[20] NREL 2004 Reference Solar Spectral Irradiance: ASTM G-173

[21] Ould Saad Hamady S and Fressengeas N 2018 EPJ Photovoltaics 913

[22] Barkhouse D, Haight R, Sakai N, Hiroi H, Sugimoto H and Mitzi D 2012 Applied Physics Letters 100193904

[23] Nguyen M, Ernits K, Tai K F, Ng C, Pramana S, Sasangka W, Batabyal S, Holopainen T, Meissner D and Neisser A 2015 Solar Energy 111 344-349

[24] Walsh A, Chen S, Wei S and Gong X 2012 Advanced Energy Materials 2 400-409

[25] Shin B, Gunawan O, Zhu Y, Bojarczuk N, Chey S and Guha S 2013 Progress in Photovoltaics: Research and Applications 21 72-76 